\title{
Neurogenesis in the Normal Ageing Hippocampus: A Mini-Review
}

\author{
Désirée R.M. Seib Ana Martin-Villalba \\ Division of Molecular Neurobiology, German Cancer Research Center (DKFZ), DKFZ-ZMBH Alliance, \\ Heidelberg, Germany
}

\section{Key Words}

Neurogenesis · Ageing · Hippocampus · Memory

\begin{abstract}
New neurons are continuously generated and added to neural circuits in the adult brain. However, increasing age imposes changes in neural progenitor cells and their microenvironment that lead to a reduction of neurogenesis. Agerelated decreased production of new neurons in the neurogenic dentate gyrus has been associated with memory impairments. Several mechanisms are known that might counteract this decline in cognitive functions. Here, we give an overview of ageing-related changes in neurogenesis in the brain of humans and rodents. We discuss possible causes for reduced neurogenesis with age, its consequences on cognition, and how neurogenesis might be restored in old age.

๑) 2014 S. Karger AG, Basel
\end{abstract}

\section{Introduction}

Neurogenesis describes the process of the generation, migration, maturation, and functional integration of newborn neurons into the pre-existing neuronal network. Neural progenitor cells (NPCs) activated to produce neurons have been identified in the subgranular zone (SGZ) of the dentate gyrus (DG) of the hippocam-
(C) 2014 S. Karger AG, Basel

0304-324X/14/0614-0327\$39.50/0 pus and the subventricular zone (SVZ) of the lateral ventricles in rodents, primates, and humans [1-5]. In the SVZ, NPCs produce new neurons for the olfactory bulb and the striatum, whereby SVZ neurogenesis is crucial for olfaction in rodents and a decrease in striatal neurogenesis in humans has been implicated in Huntington's disease $[4,6]$. Hippocampal neurogenesis is involved in learning, memory, and emotional behaviour. Neurogenesis has been reported to decrease with age in humans and rodents with distinct dynamics $[4,5,7]$. However, the extent, causes, and functional consequences of the age-related decline in neurogenesis are not fully understood.

This mini-review primarily focuses on neurogenesis in the rodent hippocampus during ageing, factors that play a role in regulating neurogenesis with age, and potential drawbacks associated with a decrease of neurogenesis during ageing. For in depth reviews, see Christian et al. [6], Fuentealba et al. [8], and Lee et al. [9]. Furthermore, we will discuss the potential of targeted therapies to increase neurogenesis in ageing.

\section{Adult Neurogenesis}

SVZ and SGZ NPCs in the adult brain are able to generate neurons and glia not only during development but also in adulthood [6]. The ability of SGZ NPCs to generate neurons is tightly regulated by factors within the neurogenic niche. Additionally, the close contact with blood

\section{KARGER 125}

E-Mail karger@karger.com www.karger.com/ger
Ana Martin-Villalba

Division of Molecular Neurobiology

German Cancer Research Center (DKFZ), DKFZ-ZMBH Alliance DE-69120 Heidelberg (Germany)

E-Mail a.martin-villalba@ dkfz-heidelberg.de 
vessels of SGZ NPCs enables the integration of systemic signals [8]. SGZ NPCs of the hippocampal DG can be divided into two subpopulations: the quiescent non-dividing radial glia cells and the active horizontal NPCs. Quiescent radial glia are able to transform into horizontal NPCs and proliferate [10]. Both radial and horizontal NPCs express GFAP (glial fibrillary acidic protein) and nestin. An in vivo clonal analysis study has shown that NPCs can either divide symmetrically in order to selfrenew or divide asymmetrically in order to generate more restricted NPCs [11]. These NPCs, which are restricted to the neuronal lineage, exhibit an increased proliferative potential, are positive for Tbr2 (a T-box transcription factor 2), and express proliferation markers such as MCM2 [6]. After several rounds of divisions, NPCs transform into immature neurons, which express doublecortin (DCX) and PSA-NCAM (polysialated neural cell adhesion molecule). Immature neurons are mainly post-mitotic and migrate into the DG granule cell layer (GCL) where they differentiate into mature neurons. Mature glutamatergic neurons in the GCL of the DG have formed spines in the molecular layer of the hippocampus where they receive input from the entorhinal cortex (EC). Furthermore, they extend their axons via the mossy fibre pathway to the CA3 region of the hippocampal Ammon's horn (CA) and the hilus and they also target inhibitory interneurons in the GCL.

\section{The Importance of Adult-Born Neurons}

In the hippocampus, the developmental age at which neurons are generated and the maturation stage of the newborn neuron determine their mode of activation within the neuronal network and, therefore, their function in learning and memory $[12,13]$. Neurons born during early postnatal life or born in adulthood are activated under different behavioural paradigms. In detail, developmentally born neurons are activated in a different environment, whereas adult-born neurons are crucial for spatial problem solving, i.e. they are activated by learning a new behavioural paradigm. Such differences might be explained by distinct electrophysiological properties of newborn neurons as compared to their mature counterparts [6].

Already 3-day-old neurons are tonically activated by ambient GABA ( $\gamma$-aminobutyric acid), whereas GABAmediated synaptic inputs from local interneurons are received by 7-day-old neurons. Within the first 2 weeks, GABA depolarizes newborn cells [6]. This input is crucial for survival and proper integration into the neuronal network. When neurons mature further, GABA hyperpolarizes newborn neurons just like mature granule cells and they start to receive glutamatergic inputs from the EC. Even if they are integrated into the network as mature neurons are, they are still distinct from mature granule cells due to their higher membrane resistance and high resting potentials. Four-week-old newborn neurons show increased long-term potentiation compared to mature cells, which is mediated by NR2B-containing NMDA receptors [14]. They also show enhanced activation compared to mature granule cells, which, in turn, leads to increased activation of CA3 neurons $[12,15]$. These properties confer the ability of newborn neurons to become activated at a lower activity threshold in distinct contexts than the thresholds required for activation of mature granule neurons. Yet, not only their electrophysiological properties distinguish immature neurons from mature granule cells, but also their synaptic input is different. Whereas developmentally born granule cells receive input from the medial and lateral EC, adult-born neurons are reported to be exclusively innervated by the lateral EC [16].

Immature neurons extend their axons, i.e. mossy fibres, to the CA3 region and the hilus. They target inhibitory interneurons in the GCL, hilus, and CA3 area [17]. Additional targets are excitatory hilar mossy cells and CA3 pyramidal neurons. Newborn cells form mossy fibre boutons already at 17 days of age in the CA3 and hilus region. At this stage, newborn neurons are considered functionally relevant to the neuronal network. Their synaptic boutons become increasingly larger in the CA3 region at 4 weeks of age and are indistinguishable from mature granule cells at 2.5 months of age. At 3 months of age, they have formed functional glutamatergic synapses with target cells.

The duration of neuronal maturation differs between species. Furthermore, the position of a newborn cell along the septo-temporal axis of the hippocampus impacts the speed of its maturation, as the activity of the surrounding network does [2].

Neurogenesis has been shown to be important for some types of memory in the rat such as trace fear conditioning and spatial learning in the Morris Water Maze (MWM) [18]. Levels of neurogenesis in rats and mice correlate with levels of spatial learning and memory retrieval in the MWM $[19,20]$. Furthermore, individual performance of aged rats in this task is correlated with levels of neurogenesis [19]. Similarly, increasing neurogenesis caused by running in aged mice can ameliorate an age- 
induced deficit in spatial learning and short-term retrieval of spatial memories in the MWM [20]. Vice versa, hippocampal-dependent learning tasks can increase neurogenesis in the rat [21].

A summary of multiple studies investigating the interplay between neurogenesis, learning, and memory can be found in a review by Marin-Burgin and Schinder [18]. Recent studies support the idea that neurogenesis in the DG is involved in a computational processes called pattern separation. Pattern separation describes the process in which neural circuits distinguish between two very similar experiences and encode them as distinct neuronal representations.

In detail, the DG receives partially overlapping information from its entorhinal inputs. Information is processed in the DG, which contains several times more neurons than the $\mathrm{EC}$ or the $\mathrm{CA} 3$ region. Information is passed on to the CA3 region via mossy fibre projections, which are relatively sparse compared to other cortical connections. These sparse projections generate de-correlated activity patterns in the $\mathrm{CA} 3$ region.

It is not clear yet if neurogenesis due to an increased excitability of newborn neurons supports the pattern separation process or if it rather decreases the DG's pattern separation function, i.e. if it rather generalizes input and performs pattern completion, the process of complementing memory traces to restore the initial memory [22].

Increasing the survival of newborn neurons in a transgenic mouse model is sufficient to increase the discrimination of two very similar contexts in a fear-conditioning paradigm [1]. On the other hand, a lack of neurogenesis impairs the discrimination of very similar objects and, furthermore, impacts spatial memory in mice when two locations that have to be distinguished are very close in space [16].

Electrophysiological recordings of rat dentate granule neurons support the DG's role in pattern separation, whereas CA3 neurons function in pattern completion, the ability to retrieve a memory from a partial input [23]. Four-week-old neurons, which are functionally integrated into the hippocampus, are crucial for memory retrieval of mice in the MWM [12]. Newborn neurons also play an important role in spatial working memory and memory consolidation but not in spatial learning in mice [1].

Neurogenesis has been recently shown to be not only involved in learning and memory, but also to be necessary to forget established memories [24]. Neuronal turnover affects local circuits in the hippocampus. If old neurons are replaced, does that mean that established memories are lost? If this was true, neuronal turnover would mean memory turnover. The question is: are certain memories stored in specific neurons? What if these neurons die; do we lose the memories they stored?

Akers et al. [24] used mice with increased neurogenesis, such as infants or adult mice with access to a running wheel, to induce increased extinction of contextual fearconditioning memories. Thus, increased neurogenesis led to increased forgetting of memories. On the contrary, acquired memories were stable if neurogenesis was depleted.

Neurogenesis also plays an important role in mood regulation. The dorsal hippocampus is believed to be important for memory formation and retrieval, whereas its ventral part is essential for emotions [25]. Neurogenesis facilitates the effect of several antidepressants, is necessary for certain antidepressants to function, and buffers stress-induced depressive-like behaviour in mice $[25,26]$. Blocking hippocampal neurogenesis by $\mathrm{X}$-irradiation can abrogate the beneficial effects of antidepressants [25]. Moreover, it has been shown that some mechanisms of antidepressant actions in behavioural tests are neurogenesis dependent, whereas others are neurogenesis independent [27]. Increasing neurogenesis with a combination of a genetic approach and exercise could also reduce anxiolytic behaviour [1]. Stress and the resulting release of glucocorticoids have been shown to decrease neurogenesis in rodents and primates [25]. Along this line, magnetic resonance imaging (MRI) studies indicate that depressed human patients show a reduction in their hippocampal size [28]. Neurogenesis in mice can counteract a stress-induced glucocorticoid increase and stress-induced depressive-like behaviour [26].

\section{Neurogenesis in the Ageing Human Brain}

Frisén and colleagues [4,5] performed birth-dating studies of single neurons in the human brain at various ages. Nuclear-bomb test-derived $14 \mathrm{C}$ was measured in neurons' nuclear DNA and compared to atmospheric $14 \mathrm{C}$ levels relative to the birth date of the human being in order to determine the time of birth of a neuron $[4,5]$. With this method, the age of neurons in the olfactory bulb was dated back to embryogenesis or early postnatal stages of a human being [4]. By contrast, active NPCs within the SVZ of adult humans supply the adult striatum with interneurons [4]. These findings suggest that immature neurons migrate from the SVZ to the olfactory bulb in humans only at embryonic and very young ages (before 
18 months of age) [29]; however, SVZ-derived neurons are added to the striatum throughout adult life in humans [4].

Similarly, neurogenesis in the hippocampus is ongoing in adult and aged human individuals, exhibiting a moderate age-related decrease [5]. Nevertheless, since the number of newborn neurons is rather small compared to the number of mature granule cells, a moderate decrease in newborn cells might also impact the system. Thereby, the decline in neurogenesis is non-linear and the highest decease happens at young ages. It is of note that the neuronal turnover in the hippocampus seems to be higher in humans than it is in mice. The cycling population in hippocampal neurons lies at approximately $35 \%$ in humans, whereas the DG cycling population is about $10 \%$ in mice.

Post mortem studies in humans have reported an even higher decrease of neurogenesis during ageing [30]. However, in very old individuals, DCX-positive progenitors were still present. Furthermore, antidepressant treatment increases NPC proliferation even in aged humans [31].

In addition to the $14 \mathrm{C}$ method and post mortem studies, MRI detecting cerebral blood flow has been used to indirectly examine neurogenesis in humans. Cerebral blood flow reflects DG activity as well as angiogenesis, which in several species correlate with hippocampal neurogenesis [31, 32]. Additionally, hippocampal function measured with MRI is correlated with memory decline, although a solid causal link of observed changes to memory decline is missing in the study by Small et al. [33].

Neurogenesis is deregulated in various human brain pathologies and neurodegenerative diseases such as Alzheimer's, Parkinson's, and Huntington's disease. For further details on rodent and human studies, see the review by Winner et al. [34].

\section{Neurogenesis in the Ageing Rodent Brain}

The rodent DG contains a constant number of granule cells and, thus, a defined overall size over the animal's lifetime [7]. Cell death in the dentate GCL peaks at 2 months of age in mice and decreases thereafter, pointing towards a regulated granule cell homeostasis. In contrast, the production of immature neurons decreases drastically with age [1]. In mice, the number of dividing cells decreases exponentially between the age of 1 and 9 months of life [7]. Thereafter, mathematical modelling indicates that it decreases with a non-constant rate until it is barely present in 18-month-old mice [35]. It is not yet completely understood what causes the decline in neurogenesis with age. Several theories exist to explain this decrease, ranging from changes in the intrinsic state of NPCs or the local microenvironment to the levels of systemic factors.

One possibility is that only the number of actively dividing progenitor cells, which belong to the horizontal NPC population, decreases with age, whereas the number of quiescent radial glia-like NPCs remains constant. The fact that certain stimuli are able to increase neurogenesis in aged mice suggests that a population of quiescent NPCs that has the potential to become activated is still present at old age [10]. NPCs and immature neurons disappear in mice over time because active NPCs transform into astrocytes after a phase of actively producing NPCs $[1,10,11]$. In detail, NPCs are able to generate a certain number of neuronal progenitors that continue to proliferate and generate immature neurons. Thereafter, they transform into non-proliferating astrocytes [1]. This terminal astrocytic differentiation leads to a decrease in NPC numbers with increasing age. Similarly, tracing single NPC clones over time shows that NPCs are removed from their own cell clones by differentiating into neurons or astrocytes [11]. These observations explain why neurogenesis declines with age in mice, while gliogenesis does not [1].

It is a well-accepted phenomenon in rodents that a reduction in neurogenesis can impair diverse forms of learning and memory, whereas increased neurogenesis improves performance in some memory tasks [18]. It is speculated that a decrease in neurogenesis in ageing correlates with a decrease in cognitive performance in mice [36]. Increasing adult hippocampal neurogenesis in mice can lead to an improvement in memory [1]. Increasing the number of immature and mature adult-born neurons in mice is beneficial for the ability to discriminate similar contexts [1]. Furthermore, increasing the complexity and the number of newborn young neurons in mice leads to enhanced working memory and memory consolidation independent of the age of the animal [1].

The risk of developing age-related memory disorders is presumably predictable earlier in life [37]. Individuals with a lower neurogenesis at younger ages might be more vulnerable to neurogenesis-related memory loss, whereas a higher neurogenesis at younger ages might be preventive.

A variety of factors can impact neurogenesis in a positive or negative way. Social interaction, an enriched environment, physical exercise, or hippocampal-dependent learning affect neurogenesis in the DG of rodents $[1,18]$. Not only the environment, but also local niche signals and intrinsic NPC behaviour can affect levels of neurogenesis. 
In the following, we are going to elucidate changes that occur during ageing concerning systemic alterations, modifications of the neurogenic niche, and intrinsic NPC changes, focusing on our knowledge derived mainly from rodent studies.

\section{Age-Related Changes Affecting Neurogenesis}

The physiology of the body changes during ageing, including changes in the cellular immune response, inflammatory mediators, hormonal levels, and structural changes of the vasculature. Changes in multiple hormone classes have been shown to impact the ageing brain. Basal glucocorticoid levels, for example, increase with age in mice [21]. Furthermore, humans show increased hypothalamic-pituitary-adrenal (HPA) axis responsivity to stress with age, whereby a high HPA axis activity might be associated with an increase in cognitive decline [38].

Additionally, glucocorticoid and mineralocorticoid receptor expression levels are higher in the DG of aged mice compared to younger animals. Adrenalectomy, which prevents glucocorticoid release by removing the adrenal gland, can avert the age-induced decrease in adult neurogenesis, identifying increased glucocorticoid hormone levels as a cause of reduced plasticity in the ageing hippocampus [21].

Ghrelin, a growth hormone that is released by the stomach according to the nutritional status of the body, has been shown to have neuroprotective functions in mice [39]. It promotes neurogenesis and synaptic plasticity in the hippocampus. Ghrelin levels are high in the blood of young rats, whereas its abundance is reduced during ageing. Young rats that lack the ghrelin receptor GHS-R (growth hormone secretagogue receptor) exhibit reduced hippocampal neurogenesis and reduced spine density in granule cell neurons. This effect faints in middle-aged GHS-R knockout animals, which show no difference regarding neurogenesis and spine density compared to age-matched wild-type counterparts. Mathematical modelling of adult neurogenesis predicts that the effect of an altered progenitor cell parameter on the number of existing cells weakens with time due to the age-related decline of progenitor cells [35]. Thus, only induced deletion of ghrelin in old age would clarify its role in the ageing brain. Nevertheless, the observed reduction in ghrelin levels in aged animals could be a reason for reduced neurogenesis and impaired synaptic plasticity. Similarly, IGF-1 (insulin growth factor-1) and VEGF (vascular endothelial growth factor) are fac-

Neurogenesis in the Normal Ageing

Hippocampus tors that decrease with ageing in rats and could contribute to a decrease of hippocampal neurogenesis in old age [9].

Not only can growth factors reach NPCs via the vasculature, but also immunomodulatory factors do so [37]. For example, LPS-induced inflammation decreases hippocampal neurogenesis in young animals and non-steroidal anti-inflammatory drugs can rescue this phenotype. Inflammation can also negatively impact integration of immature neurons into the hippocampal network in mice and rats. It is now recognized that ageing is characterized by a mild pro-inflammatory state. Elevated circulating levels of inflammatory factors in old age have negative effects on neurogenesis [37]. Another bloodborne factor that reduces adult hippocampal neurogenesis is the chemokine CCL11 [chemokine (C-C motif) ligand 11] [40]. CCL11 levels increase in the plasma of aged mice and treatment of young mice with CCL11 leads to a reduction in hippocampal neurogenesis. Furthermore, CCL11 levels are elevated in human plasma and cerebrospinal fluid, indicating its significance in neurogenesis in aged humans.

The brain vasculature is also affected by ageing [9]. Mechanical and structural properties of blood vessels change and, as a consequence, they become more permeable for proteins that would be excluded from the brain by the blood-brain barrier in younger rodents. These proteins might initiate an inflammatory response in the aged brain.

Not only the supply of NPCs with systemic factors is affected by ageing, but also local neighbouring cells change with age and growth factor supply becomes insufficient [9]. In healthy young rodents, astrocytes provide immature neurons with FGF-2 (fibroblast growth factor-2). In aged animals, astrocytes become activated at a higher rate than in young animals and the resulting reactive astrocytes produce less FGF-2, meaning less support for neuronal maturation. Like NPCs, astrocytes are in close contact to the vasculature via their astrocytic endfeet that enable sensing blood signals and a response to age-related systemic changes.

Notch signalling is another important regulator of adult neurogenesis, which is altered during ageing. Notch-responsive NPCs become quiescent during ageing and stop producing new neurons. However, these quiescent NPCs can become reactivated to produce neurons by stimuli such as exercise even in aged mice [10].

Wnt signalling plays an important role in NPC proliferation [8]. A population of specialized astrocytes located in the DG SGZ of mice produces Wnt3a, which promotes 
neuronal differentiation. Downregulation of this factor in old age negatively affects neurogenesis.

Microglia, the resident immune cells of the brain, are another source of local factors that can influence NPC fate $[9,37]$. Activated microglia can have adverse effects on neurogenesis by either producing reactive oxygen species (ROS) or by releasing inflammatory cytokines. ROS might be produced by the removal of cell debris, whereas cytokines might be released due to systemic changes such as inflammation or ageing. Predominant cytokines released by activated microglia are TNF- $\alpha$ (tumour necrosis factor- $\alpha$ ), IL-1 $\beta$ (interleukin $1 \beta$ ), and IL-6 (interleukin 6). These cytokines can inhibit neurogenic differentiation of NPCs and favour the production of astrocytes. Beneficial growth factors that can also be released by microglia are IGF-1 and BDNF (brain-derived neurotrophic factor).

Microglia can also receive and become activated by signals from neighbouring neurons [37]. This neuronmicroglia crosstalk is affected by ageing in rodents. Damaged neurons, for example, can release chemokines or glutamate, which activate microglia. Fractalkine (FKN), also called CX3CL1, is a neuroimmunoregulatory protein that is released from healthy neurons. FKN keeps microglia in an inactive state. Its expression decreases with age in the hippocampus, which leads to an increased activation of microglia. Reconstituting FKN in aged rats is able to increase neurogenesis. Conversely, blocking the FKN receptor CX3CR1 in young animals reduces hippocampal neurogenesis. This mechanism is mediated through IL$1 \beta$. Other molecules that increase in old age and are released by neurons to keep microglia inactive are CD200, CD47, and CD55.

The abundance of neurotransmitters and their receptors is also affected by ageing. GABA production and signalling decrease with age in the rat hippocampus [41]. Since GABA is essential for immature neuron survival and integration, this decrease might negatively impact proliferation and survival and, additionally, might lead to dendritic atrophy in the ageing DG. Acetylcholine (ACh) release is also reduced during ageing in mice [42]. A reduction in cholinergic inputs decreases proliferation, short-term survival, differentiation into mature neurons and increases apoptosis in the hippocampus in the mouse [21]. Dopaminergic projections from the ventral tegmental area innervate the DG. Dopamine increases hippocampal rat neurogenesis and, vice versa, its depletion decreases NPC proliferation [27]. VTA dopaminergic neurons and hippocampal dopamine receptor 1 (DR1) and DR2 decrease with age in mice [43].
Ageing severely affects proliferation of NPCs and survival of immature neurons, leading to a reduced number of neurons integrating into the neuronal network in rodents [9]. Importantly, even if the maturation of newborn neurons may take longer in aged rats, the surviving ones are fully functional and show immediate early gene expression when being activated [44].

Only severe forms of neurodegenerative diseases decrease the number of existing DG neurons, whereas this is not the case during normal human ageing [36]. However, loss of synaptic contacts and dendritic plasticity does happen during ageing in the hippocampus. Immature neurons are one additional form of plasticity in the hippocampus that is lost during ageing. In the following, we are going to discuss intrinsic NPC changes that might lead to this decreased production of newborn neurons, their delayed maturation and decreased survival in old age.

Telomerase, an enzyme that maintains chromosome ends to ensure genomic stability, is expressed in embryonic stem cells and in some adult tissue-specific stem cells. Mouse NPCs have been shown to express telomerase [45]. However, during the ageing process, telomerase activity is reduced, resulting in telomere shortening. Short telomere length has been associated with reduced neuronal differentiation. Furthermore, immature neurons harbouring damaged telomeres undergo increased apoptosis [9].

Epigenetic changes in niche cells or NPCs themselves are another factor that contributes to a decrease in neurogenesis [46]. DNA methylation has also been suggested to be a cell-intrinsic mechanism to change fate potential in ageing NPCs shifting from a neurogenic to a gliogenic fate. Environmental stimuli such as running are able to reverse DNA methylation of growth factor genes. One important epigenetic regulator of adult NPC self-renewal is BMI1. BMI1 controls methylation of the $p 16^{\mathrm{INK} 4 \mathrm{a}}$ and p19 $9^{\mathrm{ARF}}$ gene loci, whose gene products are important factors regulating the cell cycle, senescence, and apoptosis. Importantly, expression of $p 16^{\mathrm{INK} 4 \mathrm{a}}$ and $p 19^{\mathrm{ARF}}$ increases with age in various stem cell niches. BMI1 functions as part of the PRC1 (polycomb repressor complex 1). In young animals, the PRC1 maintains the $p 16^{\mathrm{INK} 4 \mathrm{a}}$ and $p 19^{\mathrm{ARF}}$ locus in a repressed methylation state, which prevents its gene expression and allows stem cell self-renewal. During ageing, BMI1 activity decreases, leading to a loss of repressive histone marks, expression of $p 16^{\mathrm{INK} 4 \mathrm{a}}$ and $p 19^{\mathrm{ARF}}$, and, finally, stem cell senescence.

Increased DNA methylation of growth factors like Wnt ligands occurs in old reactive astrocytes. In addi- 
tion, reduced Wnt signalling in the aged DG leads to decreased survivin expression in mouse NPCs, an intracellular signalling component of the Wnt pathway [47]. This decrease leads to impaired cell cycle progression and increased quiescence of NPCs in old animals. Likewise, the expression of the Wnt antagonist Dkk1 (Dickkopf-1) increases in old NPCs. Mice that lack Dkk1 show increased NPC self-renewal and increases in the dendrite complexity of newborn neurons [1]. Importantly, Dkk1 mutants show increased neurogenesis even in 2-year-old animals, which is not counteracted by the depletion of the NPC pool with age. By contrast, loss of PTEN or GABA in mice facilitated the production of a higher number of NPCs at a young age, but this effect was counteracted by the age-related depletion of the NPC compartment [11]. Thus, Dkk1 is one factor that is responsible for reduced neurogenesis in old age and its absence does not go along with a depletion of the NPC compartment in old age.

\section{Restoring Neurogenesis in Old Age}

Hippocampal neurogenesis is an important mechanism mammals use to adapt to a changing environment. These changes might include managing novel situations, learning, and coping with stress or anxiety. First, we have to understand why neurogenesis changes during ageing. If we know the factors that cause this change, we might be able to find out how we can manipulate the system to prevent the observed age-related decline.

Hormones are an important component of the ageing organism. Discovering why hormone production is influenced by age and which effect hormonal reconstitution has on the body may lead to the identification of novel clinical targets to counteract age-related reduced production of neurons [8]. Another option is to target inflammation. Several local and systemic inflammatory molecules that are upregulated in old age have been shown to reduce neurogenesis. Reducing inflammation could restore neurogenesis and increase synaptic plasticity in the aged mouse hippocampus [40].

Running is a known paradigm that is able to increase hippocampal neurogenesis. However, the existence of a causal link between voluntary exercise and neurogenesis is heavily debated. Changes in bloodstream hormone and growth factor levels are among the more frequently supported links between effects of exercise and neurogenesis. As mentioned earlier, NPCs are in close contact to the vasculature and are, thereby, able to respond to changes

Neurogenesis in the Normal Ageing

Hippocampus in the availability of blood-borne factors [8]. Growth factors such as BDNF and NGF (nerve growth factor) have been shown to decrease with age in humans and rats, respectively $[48,49]$. Running and environmental enrichment in rodents lead to an elevated production of BDNF, GDNF (glial-derived neurotrophic factor), and NGF, amongst others [9]. In addition, exercise leads to an increased cerebral blood flow and angiogenesis and affects blood-brain barrier permeability to increase accessibility of NPCs to blood-derived growth factors, such as VEGF. Voluntary running and environmental enrichment in rodents have both been reported to increase neurogenesis; however, running has been shown to rather affect progenitor cell proliferation, whereas environmental enrichment favours survival of newborn neurons. Importantly, running can induce increased hippocampal BDNF levels, leading to increased neurogenesis even in aged animals. This supports the existence of quiescent NPCs in the hippocampus, which can be activated when they are challenged.

Exercise has the potential to increase hippocampal neurogenesis by releasing neurogenesis-promoting factors in the circulation even in very old individuals. It is a relatively uncomplicated preventive strategy which might be easily applicable in patients. A more artificial way of transferring running-related research onto human treatment would be the direct supply with running-induced growth factors into the blood stream of patients.

Chronic stress and ageing have been shown to increase Dkk1 levels in the mouse hippocampus [1]. Both scenarios are associated with reduced neurogenesis. Dkk1 exemplifies a molecule involved in stress-related depression and ageing-related memory deficits. Genetic deletion of Dkk1 could improve memory in ageing and decrease depressive-like behaviour in young animals. Thus, targeting Dkk1 with blocking antibodies, peptides, or small molecules might be one option to restore neurogenesis in these conditions.

Currently, acetylcholinesterase inhibitors are used in the clinic to treat Alzheimer's disease [50]. Since ACh decreases with age not only in mice, but also in humans, inhibiting the ACh metabolism might be a strategy to counteract the age-related cognitive decline elicited by low ACh levels. As discussed before, dopamine levels might also decrease with age; thus, Parkinson's disease medication such as L-dopa or other dopamine receptor antagonists might qualify in the future to treat cognitive decline in the ageing population [51]. So far, physical exercise in humans is the best-studied and minimally 
invasive method with the greatest regenerative potential and the least side effects [52]. Besides, exercise does not only positively affect cognition, but it is also beneficial for other age-associated diseases, such as diabetes, obesity, and heart conditions. All these effects might contribute to healthy physical and mental ageing in our increasingly ageing population. Importantly, studies show that the amount of exercise inversely correlates with the probability to develop Alzheimer's disease. As an example, 3 months of physical exercise can increase cerebral blood volume in the human DG, which has been associated with increased neurogenesis; 6 months of exercise have been shown to increase gray matter volume in the cortex. Adding mentally challenging tasks to physical therapy is even more effective. Beneficial effects due to physical and mental exercise, such as increased neuronal complexity, heightened neurotransmitter release, and more neurogenesis, lead to an increased brain plasticity, which has the potential to counteract the ageing-related mental decline in susceptible individuals.

\section{Acknowledgements}

D.R.M.S. has been funded by a postdoctoral fellowship of the German Research Foundation (DFG; 'Die Rolle von in der Entwicklung geborenen und adult geborenen granulären Nervenzellen in der Diskriminierung von Kontexten', SE 2374/1-1). A.M.-V. has received funding from the DFG (SFB873), the Federal Ministry of Education and Research (BMBF independent research group), and the German Cancer Research Center (DKFZ).

\section{Disclosure Statement}

The authors declare that they have no conflicts of interest.

\section{References}

1 Seib DR, Corsini NS, Ellwanger K, Plaas C, Mateos A, Pitzer C, Niehrs C, Celikel T, Martin-Villalba A: Loss of Dickkopf-1 restores neurogenesis in old age and counteracts cognitive decline. Cell Stem Cell 2013;12:204214.

- 2 Snyder JS, Ferrante SC, Cameron HA: Late maturation of adult-born neurons in the temporal dentate gyrus. PLoS One 2012;7:e48757.

- 3 Gould E, Reeves AJ, Fallah M, Tanapat P, Gross CG, Fuchs E: Hippocampal neurogenesis in adult Old World primates. Proc Natl Acad Sci U S A 1999;96:5263-5267.

4 Ernst A, Alkass K, Bernard S, Salehpour M, Perl S, Tisdale J, Possnert G, Druid H, Frisén J: Neurogenesis in the striatum of the adult human brain. Cell 2014;156:1072-1083.

5 Spalding KL, Bergmann O, Alkass K, Bernard S, Salehpour M, Huttner HB, Bostrom E, Westerlund I, Vial C, Buchholz BA, Possnert G, Mash DC, Druid H, Frisén J: Dynamics of hippocampal neurogenesis in adult humans. Cell 2013;153:1219-1227.

6 Christian KM, Song H, Ming GL: Functions and dysfunctions of adult hippocampal neurogenesis. Annu Rev Neurosci 2014;37:243262.

7 Ben Abdallah NM, Slomianka L, Vyssotski AL, Lipp HP: Early age-related changes in adult hippocampal neurogenesis in C57 mice. Neurobiol Aging 2010;31:151-161.

-8 Fuentealba LC, Obernier K, Alvarez-Buylla A: Adult neural stem cells bridge their niche. Cell Stem Cell 2012;10:698-708.

-9 Lee SW, Clemenson GD, Gage FH: New neurons in an aged brain. Behav Brain Res 2012; 227:497-507.

10 Lugert S, Basak O, Knuckles P, Haussler U, Fabel K, Gotz M, Haas CA, Kempermann G,
Taylor V, Giachino C: Quiescent and active hippocampal neural stem cells with distinct morphologies respond selectively to physiological and pathological stimuli and aging. Cell Stem Cell 2010;6:445-456.

11 Bonaguidi MA, Wheeler MA, Shapiro JS, Stadel RP, Sun GJ, Ming GL, Song H: In vivo clonal analysis reveals self-renewing and multipotent adult neural stem cell characteristics. Cell 2011;145:1142-1155.

12 Gu Y, Arruda-Carvalho M, Wang J, Janoschka SR, Josselyn SA, Frankland PW, Ge S: Optical controlling reveals time-dependent roles for adult-born dentate granule cells. Nat Neurosci 2012;15:1700-1706.

13 Tronel S, Lemaire V, Charrier V, Montaron MF, Abrous DN: Influence of ontogenetic age on the role of dentate granule neurons. Brain Struct Funct 2014, Epub ahead of print.

14 Ge S, Yang CH, Hsu KS, Ming GL, Song H: A critical period for enhanced synaptic plasticity in newly generated neurons of the adult brain. Neuron 2007;54:559-566.

15 Marin-Burgin A, Mongiat LA, Pardi MB, Schinder AF: Unique processing during a period of high excitation/inhibition balance in adult-born neurons. Science 2012;335:12381242.

16 Vivar C, Potter MC, Choi J, Lee JY, Stringer TP, Callaway EM, Gage FH, Suh H, van Praag $\mathrm{H}$ : Monosynaptic inputs to new neurons in the dentate gyrus. Nat Commun 2012;3:1107.

-17 Toni N, Laplagne DA, Zhao C, Lombardi G, Ribak CE, Gage FH, Schinder AF: Neurons born in the adult dentate gyrus form functional synapses with target cells. Nat Neurosci 2008;11:901-907.

18 Marin-Burgin A, Schinder AF: Requirement of adult-born neurons for hippocampus-de- pendent learning. Behav Brain Res 2012;227: 391-399.

19 Drapeau E, Mayo W, Aurousseau C, Le MM, Piazza PV, Abrous DN: Spatial memory performances of aged rats in the water maze predict levels of hippocampal neurogenesis. Proc Natl Acad Sci U S A 2003; 100:1438514390.

20 van Praag H, Shubert T, Zhao C, Gage FH: Exercise enhances learning and hippocampal neurogenesis in aged mice. J Neurosci 2005; 25:8680-8685.

21 Veena J, Rao BS, Srikumar BN: Regulation of adult neurogenesis in the hippocampus by stress, acetylcholine and dopamine. J Nat Sci Biol Med 2011;2:26-37.

22 Aimone JB, Deng W, Gage FH: Resolving new memories: a critical look at the dentate gyrus, adult neurogenesis, and pattern separation. Neuron 2011;70:589-596.

23 Neunuebel JP, Knierim JJ: CA3 retrieves coherent representations from degraded input: direct evidence for CA3 pattern completion and dentate gyrus pattern separation. Neuron 2014;81:416-427.

24 Akers KG, Martinez-Canabal A, Restivo L, Yiu AP, De Cristofaro A, Hsiang HL, Wheeler AL, Guskjolen A, Niibori Y, Shoji H, Ohira K, Richards BA, Miyakawa T, Josselyn SA, Frankland PW: Hippocampal neurogenesis regulates forgetting during adulthood and infancy. Science 2014;344:598-602.

25 Castren E, Hen R: Neuronal plasticity and antidepressant actions. Trends Neurosci 2013; 36:259-267.

26 Snyder JS, Soumier A, Brewer M, Pickel J, Cameron HA: Adult hippocampal neurogenesis buffers stress responses and depressive behaviour. Nature 2011;476:458-461. 
27 Doze VA, Perez DM: G-protein-coupled receptors in adult neurogenesis. Pharmacol Rev 2012;64:645-675.

28 Bremner JD, Elzinga B, Schmahl C, Vermetten E: Structural and functional plasticity of the human brain in posttraumatic stress disorder. Prog Brain Res 2008;167:171-186.

29 Sanai N, Nguyen T, Ihrie RA, Mirzadeh Z, Tsai HH, Wong M, Gupta N, Berger MS, Huang E, Garcia-Verdugo JM, Rowitch DH, Alvarez-Buylla A: Corridors of migrating neurons in the human brain and their decline during infancy. Nature 2011;478:382-386.

30 Knoth R, Singec I, Ditter M, Pantazis G, Capetian P, Meyer RP, Horvat V, Volk B, Kempermann G: Murine features of neurogenesis in the human hippocampus across the lifespan from 0 to 100 years. PLoS One 2010;5:e8809.

- 31 Sierra A, Encinas JM, Maletic-Savatic M: Adult human neurogenesis: from microscopy to magnetic resonance imaging. Front Neurosci 2011;5:47.

32 Small SA, Chawla MK, Buonocore M, Rapp $\mathrm{PR}$, Barnes CA: Imaging correlates of brain function in monkeys and rats isolates a hippocampal subregion differentially vulnerable to aging. Proc Natl Acad Sci U S A 2004;101: 7181-7186.

33 Small SA, Tsai WY, DeLaPaz R, Mayeux R, Stern Y: Imaging hippocampal function across the human life span: is memory decline normal or not? Ann Neurol 2002;51:290-295.

-34 Winner B, Kohl Z, Gage FH: Neurodegenerative disease and adult neurogenesis. Eur J Neurosci 2011;33:1139-1151.

35 Ziebell F, Martin-Villalba A, Marciniak-Czochra A: Mathematical modelling of adult hippocampal neurogenesis: effects of altered stem cell dynamics on cell counts and bromodeoxyuridine-labelled cells. J R Soc Interface 2014;11:20140144.
36 Small SA, Schobel SA, Buxton RB, Witter MP, Barnes CA: A pathophysiological framework of hippocampal dysfunction in ageing and disease. Nat Rev Neurosci 2011;12:585-601.

37 Gemma C, Bachstetter AD, Bickford PC: Neuron-microglia dialogue and hippocampal neurogenesis in the aged brain. Aging Dis 2010;1:232-244.

38 Raskind MA, Peskind ER, Wilkinson CW: Hypothalamic-pituitary-adrenal axis regulation and human aging. Ann NY Acad Sci 1994; 746:327-335.

39 Cahill S, Tuplin E, Holahan MR: Circannual changes in stress and feeding hormones and their effect on food-seeking behaviors. Front Neurosci 2013;7:140.

40 Villeda SA, Luo J, Mosher KI, Zou B, Britschgi M, Bieri G, Stan TM, Fainberg N, Ding Z, Eggel A, Lucin KM, Czirr E, Park JS, Couillard-Despres S, Aigner L, Li G, Peskind ER, Kaye JA, Quinn JF, Galasko DR, Xie XS, Rando TA, Wyss-Coray T: The ageing systemic milieu negatively regulates neurogenesis and cognitive function. Nature 2011;477:90-94.

41 Rao MS, Hattiangady B, Shetty AK: The window and mechanisms of major age-related decline in the production of new neurons within the dentate gyrus of the hippocampus. Aging Cell 2006;5:545-558.

42 Itou Y, Nochi R, Kuribayashi H, Saito Y, Hisatsune T: Cholinergic activation of hippocampal neural stem cells in aged dentate gyrus. Hippocampus 2011;21:446-459.

43 Saroja SR, Kim EJ, Shanmugasundaram B, Hoger H, Lubec G: Hippocampal monoamine receptor complex levels linked to spatial memory decline in the aging C57BL/6J. Behav Brain Res 2014;264:1-8.

44 Marrone DF, Ramirez-Amaya V, Barnes CA: Neurons generated in senescence maintain capacity for functional integration. Hippocampus 2012;22:1134-1142.
45 Jaskelioff M, Muller FL, Paik JH, Thomas E, Jiang S, Adams AC, Sahin E, Kost-Alimova M, Protopopov A, Cadinanos J, Horner JW, Maratos-Flier E, Depinho RA: Telomerase reactivation reverses tissue degeneration in aged telomerase-deficient mice. Nature 2011; 469:102-106.

46 Pollina EA, Brunet A: Epigenetic regulation of aging stem cells. Oncogene 2011;30:31053126.

47 Miranda CJ, Braun L, Jiang Y, Hester ME, Zhang L, Riolo M, Wang H, Rao M, Altura RA, Kaspar BK: Aging brain microenvironment decreases hippocampal neurogenesis through Wnt-mediated survivin signaling. Aging Cell 2012;11:542-552.

48 Erickson KI, Prakash RS, Voss MW, Chaddock L, Heo S, McLaren M, Pence BD, Martin SA, Vieira VJ, Woods JA, McAuley E, Kramer AF: Brain-derived neurotrophic factor is associated with age-related decline in hippocampal volume. J Neurosci 2010;30:53685375.

49 Larkfors L, Ebendal T, Whittemore SR, Persson $\mathrm{H}$, Hoffer B, Olson L: Decreased level of nerve growth factor (NGF) and its messenger RNA in the aged rat brain. Brain Res 1987; 427:55-60.

50 Nordberg A, Svensson AL: Cholinesterase inhibitors in the treatment of Alzheimer's disease: a comparison of tolerability and pharmacology. Drug Saf 1998;19:465-480.

51 Mercuri NB, Bernardi G: The 'magic' of L-dopa: why is it the gold standard Parkinson's disease therapy? Trends Pharmacol Sci 2005;26: 341-344.

52 Voss MW, Vivar C, Kramer AF, van Praag H: Bridging animal and human models of exercise-induced brain plasticity. Trends Cogn Sci 2013; 17:525-544. 